\title{
Negative memory healing in seconds
}

\begin{abstract}
After 35years of teaching self-help techniques, Gary Sinclair has continued to refine his work so that it is now possible to neutralize negative memories in approximately 20 seconds. He calls this latest process Soul Link. Soul Link opens the door to your heart's energy flow by using your hands as jumper cables to connect thought memory with the energy flowing through all the meridians of the body. Gary has been using Soul Link since June 2014 with $95 \%$ success for first time users in a "content free" environment. It addresses the full spectrum of memory from minor memories to the pathologic anxiety associated with PTSD. There are no long-term studies at present. Future research is warranted.
\end{abstract}

In 1997 Dr. Bruce Lipton, Author, The Biology of Belief, wrote to Gary Sinclair: "I laud your ground breaking efforts in what may prove to be the future of medicine."

Keywords: negative memory, energy, healing, PTSD, EMDR, tapping, EFT, FTT, trauma, NLP, hypnosis, soul link
Volume 3 Issue I - 2016

Gary Sinclair

Celebrate Life, USA

Correspondence: Gary Sinclair, Cyberphysiologist, Celebrate Life, 790 Morro Road, Fallbrook, CA, USA, Tel 760-586-2828, Email celbr8life@aol.com

Received: September 08, 2015 | Published: January 19, 2016
Abbreviations: EMDR, eye movement desensitization and reprocessing; EFT, emotional freedom technique; FTT, field thought therapy; PTSD, post traumatic stress disorder; NLP, neuro linguistic programming; IHM, institute of heartmath research center; NET-CE, continuing education for healthcare professionals

\section{Introduction}

Premise 1: A person's Soul is the source of the most subtle energies of their being. From our cellular structure to our awareness of self, it is this Soul's energy that gives form to everything that makes us human beings. Thus, your body is not the real you. Your body is merely an organ of perception.

Premise 2: Spirit is the all-pervasive intelligence that engineers all of creation.

Premise 3: Soul is the manifestation of Spirit on a personal level. Soul and Spirit are intimately linked. When Soul and Spirit are disconnected human beings experience dis-ease. The heart can be used as the mechanism to reconnect the Soul with the Spirit.

\section{Gary's Story}

Born in 1945 with limited lung capacity, at an early age, Gary Sinclair became intimately acquainted with the medical profession. At age 22 he was diagnosed with Multiple Sclerosis and faced life in a wheel chair by age 34 . As the severity of the disability increased and his lung capacity diminished Gary knew he had to pursue something more than the traditional allopathic model. In 18 months using energetic therapies, like Chiropractic, Acupuncture, massage. etc., Gary was able to move on with a new life. During that time, he knew already he was developing processes that could be used to help others heal energetically. In 1987, with greater understanding of "living life as energy," Gary became the National Senior Olympic Free-Style Figure Skating Champion for the USA.

Gary has studied human energy theory while training and certifying in many modalities like Hypnosis, Hypnotherapy, Transpersonal Hypnotherapy, NLP, and therapies like Regression, Wounded Child, Soul Retrieval, Touch For Health, Healing Touch, Kinesiology, the Callahan Technique, Thought Field Therapy, Tapping, Core Transformation, Ericksonian Hypnotherapy, Psy-K, Sedona Method,
Voice-Bio Analysis, and many more. Having already healed, Gary was always asking himself, "What healing happened? When? and, Why?" This all improved his knowledge and understanding of human energy theory as he constantly asked himself, "What do we not know about "life as energy" and where can we make progress?"

He studied the work of Dr. Leonard Ravitz at William and Mary University who in 1959 showed that the Human Energy Field fluctuates with a person's mental and psychological stability. Dr. Ravitz suggested that there is a field of energy associated with the thought process. ${ }^{1}$ Dr. Caroline Myss added to that understanding explaining that your physical body is surrounded by an energy field that extends as far out as your outstretched arms and the full length of your body. It is both an information center and a highly sensitive perceptual system. .... It surrounds us and carries within it the emotional energy created by our internal and external experiences. ${ }^{2}$

Soul Link was created with these understandings as Gary wished to ensure the process used the entire energy field all at once rather than focusing on only a part of the energy structure. He understood that every memory must have some form and shape of containment within the human energy field, and that altering that containment of energy would create life changing results. Like a drop of ink in a gallon of water, the larger energy source dissipated the drop. Gary understood that any long period of time thinking about a memory only created a re-imprinting of that memory, so it had to work quickly.

Enid Hoffman writes that all energy contained in memories is feeling energy. . . . The feeling aroused by the event is registered in the memory bank along with the event. ... These unfilled expressions of our feelings, stored in memory events, express themselves in our behavior and our reactions to current events. ${ }^{3}$

Soul Link was created to neutralize the negative emotions and feelings energy associated with the memory, while generally leaving the remembrance of the memory as a historical learning experience from which to grow.

The more Gary studied energy healing, the more he understood the relationship between positive and negative energy as it relates to the holding structure of memories along with their anchoring ability to produce wellness and potentially the opposite of dis-ease. Right from the beginning, at age 36 he found ways to use human energy theory to 
neutralize minor memories and, to some degree, major memories. He worked with and continued to improve his concepts while developing other ways to improve life based on the framework of using the entire energy field of the body. A pivotal time in his quest was June 6, 2014, as he discovered how to neutralize dis-ease holding memories at their strongest levels in approximately 20seconds. That process was later given the name Soul Link and became pivotal to other changes made to enhance his two day program, Restoration and improve all of his results.

Soul Link works because it allows a person to channel connective life force energy through their own bodies using their hands. With each heartbeat, unconditional love is applied to the negative memory to which they are paying attention through feelings or emotions found within that chosen memory. You will often hear Gary say, "Love pulls whatever it touches toward its highest potential!" Soul Link works because of this truth. The positive, whole energy we think of in human energy theory is greater than the sum of the negative parts on which it is working. Because it uses the entire energy field of your body and that life sustaining energy is being refreshed with each new heartbeat, Gary would say "You love yourself to Life!" By linking the Soul connected energy to the Spirit connection through the heart, the person finds the "power verses force" principal to neutralize negative thoughts and emotions in seconds.

There are 737 references to love in the Bible. ${ }^{4}$ Love is the connective tissue of the universe, the stuff of which we are made. Love is the experience of being whole and connected to the Universe. The human heart is the way to access this love and life giving gift of Spirit.

\section{Healing heart}

At the Institute of HeartMath (IHM) Research Center, they are exploring the physiological mechanisms by which the heart communicates with the brain, thereby influencing information processing, perceptions, emotions, and health. The heart is the first organ to function when we are in utero. It is an important intelligence center along with the brain. According to Rollin McCraty, Director of Research at the Institute of HeartMath and author of the book, The Energetic Heart, the heart's electromagnetic field is about 5,000times stronger than that of the cranial brain as it interacts with and permeates every cell of our bodies. McCraty's work explains how the heart carries out the bioelectromagnetic interactions within and between people. ${ }^{5}$ Research from the HeartMath Institute has also found the intelligence of the heart to receive and respond to intuitive information. ${ }^{6}$

The heart, as a process, is important to the success of Soul Link. Conventional science has taught us that the main role of the heart is to pump blood to all the systems of our body. Thanks to the landmark research done at The Institute of HeartMath, we can now say that besides pumping blood, the heart also has an intelligence of its own. For centuries, the heart has been considered the source of emotions, courage, and wisdom. They observed that the heart was acting as though it had a mind of its own and was profoundly influencing the way we perceive and respond to the world including "shifts in perception." In essence, it appeared that the heart was affecting intelligence and awareness. Soul Link uses this influencer and recognizes definite shifts in perception take place when used properly as trained (Figure 1).

\section{Comparative works}

The hands have been used as emitters of energy since the art of healing began. For thousands of years, China and other cultures have used hands-on-healing to treat many conditions. In 1778 Franz Mesmer was the first person to write that he believed the most active points for healing the body flow through the hands. This is why therapies like Therapeutic Touch and Healing Touch, which are used in many hospitals, are shown to be that affective. Soul Link will add additional value to hospital personnel as they are trained in other uses of Soul Link like quick pain reduction and calming agitated patients.

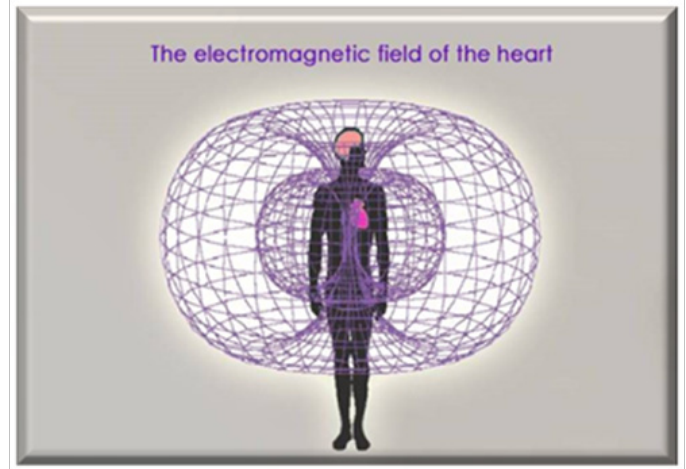

Figure I The electromagnetic field of the heart

We know of the existence of the human body's electromagnetic energy field because of high tech equipment. The SQUID, EMG, and others have been used to measure the signals. These devices not only detect the presence of such energies, but can show how they alter subtle energy fields through the application of touch. John White, in his book Future Science, lists 97 different cultures that refer to the auric phenomena with 97 different names.$^{7}$ Chinese and Korean hand acupuncture points make us aware that all of the energy fields in the body can be activated through touch. Jiro Maurai's research in Jin Shin Jyutsu ultimately revealed that each one of our fingers affects 14,400 functions within the body. ${ }^{8}$ Your nervous system is a phenomenally sensitive thirty-seven mile-long antenna that reverberates to the subtle, and not so subtle, energies of the world in which we live. ${ }^{9}$

By linking heart and hands, the life supplying energy field generated by the heart, has the ability to accesses the power availability of the combined full body energy meridian's system. When you tie this to the presence of any awareness of a memory, Soul Link powerfully unlocks the human potential as a stronger positive energy to eradicate the negative emotions and feelings in the memory in seconds. It is important that we understand, the less time a person has to think about the negative, the easier it is not prevent it from being re-imprinted over and over again (Figures 2-11).

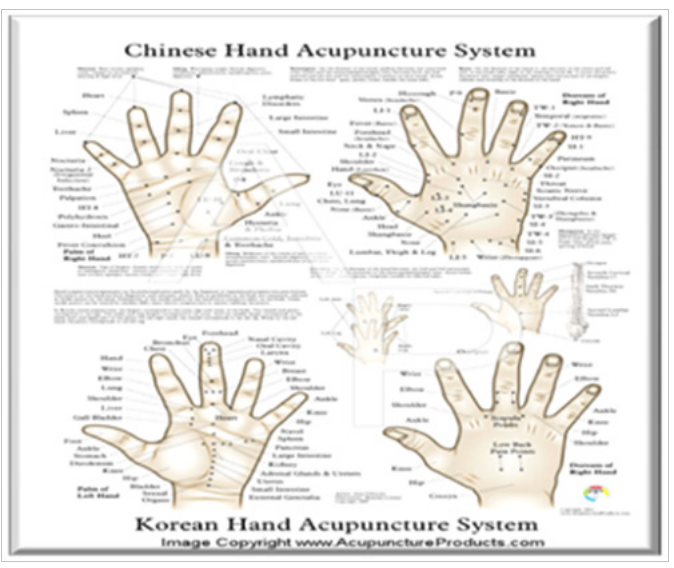

Figure 2 Acupuncture systems. 


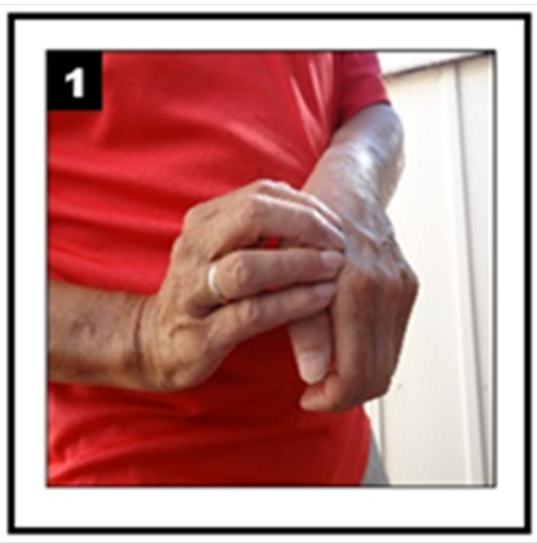

Figure 3 Place your relaxed right hand finger-tips of your fingers up against your curved and relaxed left hand. To do this, your little finger will be aimed straight at the row of knuckles on the top middle of your left hand. This location is the beginning point of contact for each repeated round in the process.

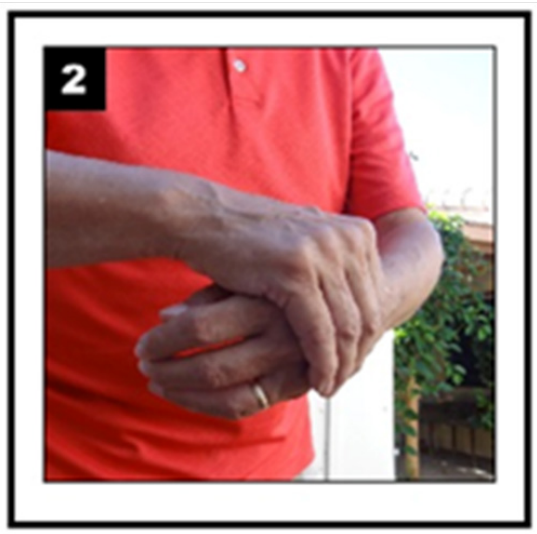

Figure 4 Once you have both hands relaxed, with your thumbs up against your fingers which are held together, begin to slide the fingers of the right hand up and over the wrist area of the left hand following the line of the knuckles with an intention to rub and feel all the area of the wrist. As you do this, note your little finger intentionally glides over the complete first row of knuckles. Your right thumb will glide close to where the wrist connects to the arm. Both hands are to make as much skin to skin surface contact as possible.

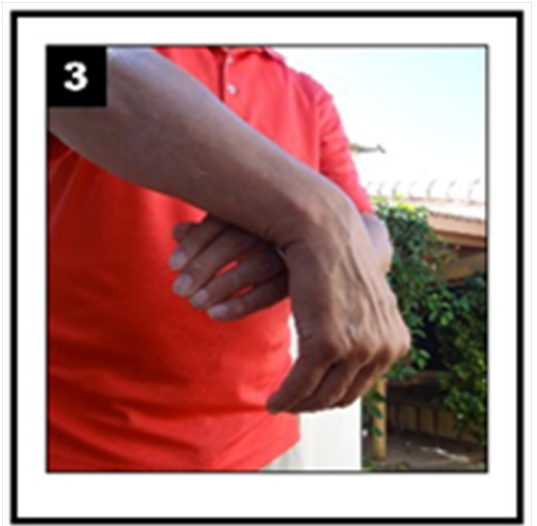

Figure 5 Allow the skin on the back of your left hand to feel all the contact of the palm of your right hand as you slide your rubbing hand. Stop when you are just past the full hand and palm area.You will have glided your entire hand all the way past the top side of your left wrist. It helps to think in terms of the knuckles, on the back of the left hand, as being the target line for the little finger to glide over.

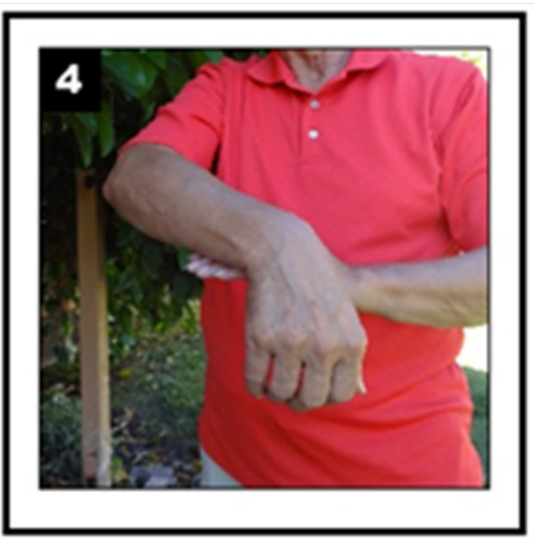

Figure 6 Next, we continue (with just a little practice) what will become a fluid movement. Slide the back of your right arm to the right until you overlay all the fingers of the left hand with your underside right arm. Your right hand palm and fingers still extend out beyond the fingers of the left hand. Stop when your right hand is in a position where, when you pull it back across, the thumb will once more glide directly over the top of the knuckles just beyond the wrist.

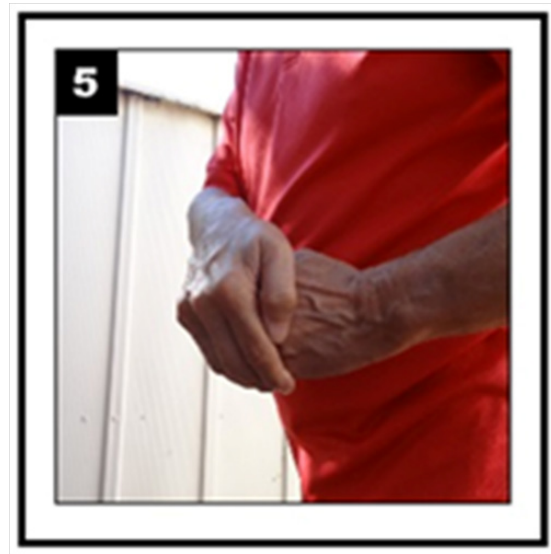

Figure 7 Begin to glide the contact surface of your right hand back across the knuckles and joints on all the fingers and ultimately the thumb of your left hand. Your hand will come all the way until the finger tips are once more at the edge of the left hand and slightly down from where they started. The best alignment coming back is to pay attention to your thumb while you bring your hand back and your thumb covers all the knuckles beyond the wrist like your little finger did earlier.

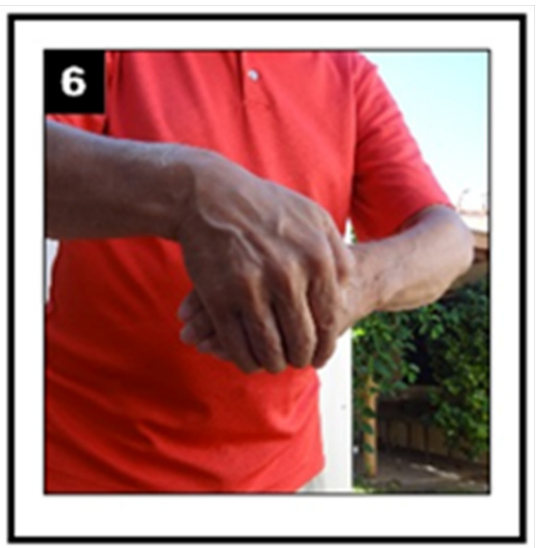

Figure 8 While that is happening, the wrist and the knuckles of your right palm hand are now rubbing all the fingers and knuckles of your left hand. 


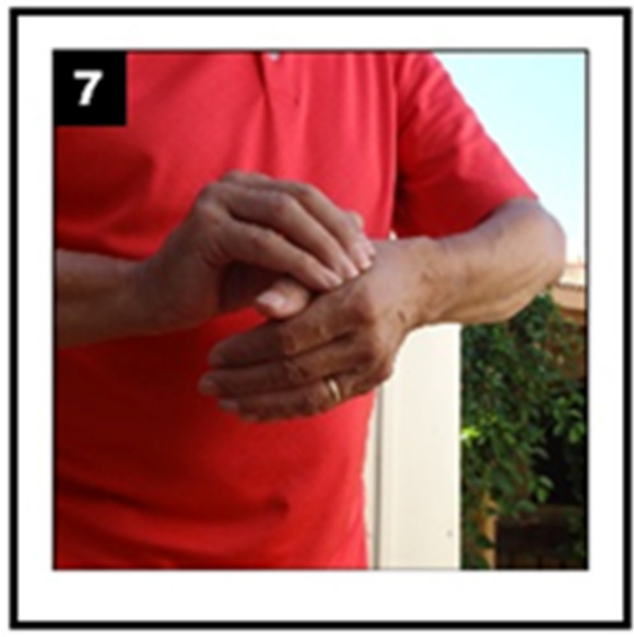

Figure 9 Everything from here is a repeat of the steps above as you slide your fingers back to the starting position.

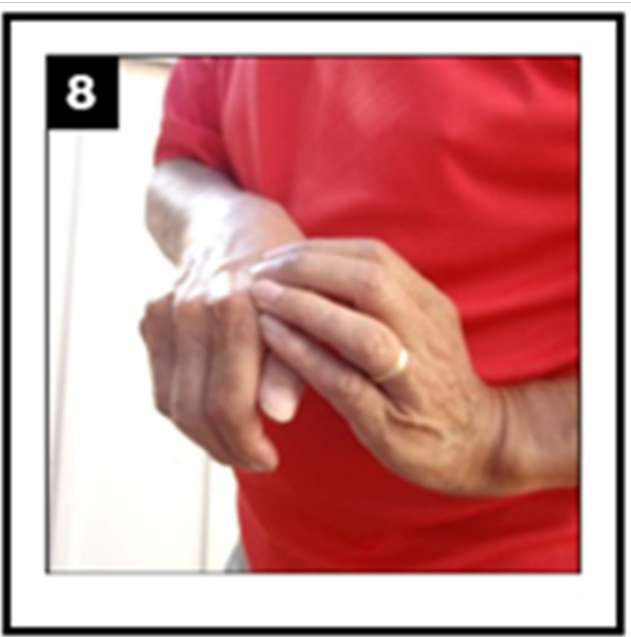

Figure I 0 Now if I put a mirror between your hands and you were ready to do the next step, you would merely repeat it on the right hand doing the reverse of your slide and glide pattern. On the left hand you went in a clockwise direction on the top of the hand making four complete patterns.

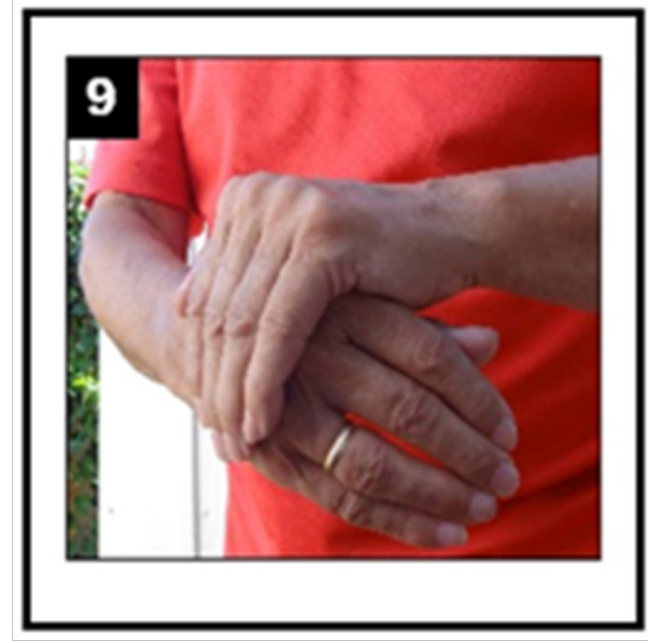

Figure I I On the right hand top, you will go in a counter-clockwise direction. Practice until you can do four more complete patterns almost without thinking.

\section{Comparative works}

In a comparative of existing works, EMDR uses the fact that the eyes are the access to the Soul, and thus uses eye movement to find the memory stored location for neutralization. Soul Link begins with the client thinking about the memory and thus connecting to a known memory location, and then uses the entire energy of the Soul in the process of neutralizing as each new heart beat replenishes the Soul's energy field. There is no need for any long discussion on what the memory is about, nor how much it bothers you or your client, once certified. Most of the work Gary personally does is in a "content free" environment, even when done to the client, rather than the client learning to do it by themselves.

In a further comparative of existing works, EFT, FTT, Tapping, and the Callahan Technique, etc. are using the existence of the energy meridians in the body to "tap" into an individual meridian. By tapping specific locations and involved meridians, the processes seek to neutralize a negative. Soul Link begins with the client using all of the energy meridians in the body at the same time through the tops and bottoms of both hands.

In short, "Soul Link" opens the door to your heart's energy flow by using your hands as jumper cables to connect thought with the energy flowing through all the meridians of the body. "It neutralizes unwanted emotions and feelings stored in the mind and body as negative memory in approximately 20 seconds.

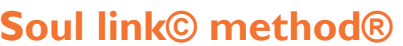

For a client to be taught to use Soul Link, a first process called The Ball is used to allow the client to know how to feel their own energy and neutralize minor memories. The Box, which is capable of handling stronger memories, is taught next. Gary Sinclair has taught these two processes for 35years as they came out of his own personal healing work. Soul Link as demonstrated here is taught next. This partial presentation is presented the way you would teach it by demonstration this part of Soul Link is presented the way you would teach it so that you might experience the power in what Soul Link offers. The protocols for doing this "TO" the client, rather than teaching them to do it themselves, will not be given here. It is however taught in Soul Link Certification. The protocols for doing this "TO" the client, rather than teaching them to do it themselves, will not be given here. It is however taught in Soul Link Certification.

When you have completely slid across, you will feel like you could drop your right hand down at the wrist joint over the left hands outside edge. Dropping the wrist is not necessary; this is only to explain how far you will go in sliding as you intentionally feel the rub across with both hands making contact.

Check by coming back to the starting position again. This time, as you slide your relaxed right hand across, you can notice how much you will feel the connection. Set your intention to make rubbing contact as you feel all the connective sensations that are possible. Be sure you used the alignment of your little finger on your right hand going over the top line of knuckles as a way of allowing the placement of the hands to make the best use of the energy contacts.

Make sure you have not straightened nor sought to extend any fingers. Both hands must stay relaxed in the complete process for the best results. Keep your thumb up against your fingers.

It is easiest NOT to move your left hand while learning the process. It should remain steady while your right hand does the clockwise 
pattern movement. Most people hold the hand being rubbed in the air and slightly in front as they look at their hands. That way you get to see the alignment of the little finger sliding over and covering the knuckles on the pass and the thumb covering the same row of knuckles coming back. The key is being "loose and relaxed" while the right hand does the clockwise rubbing rotation. You can think of it like applying a lotion all over the back of the hand. When you apply a lotion, you keep your hands relaxed. In this way you "feel" it all and that is most important.

You have now completed the Soul Link movement process for one right hand palm pass over the top left hand. Now consider that you basically have designed the action to be like a box as you go across, slide down, and come back across in a clockwise direction.

When you teach this technique, it is important that your client is able to do the steps almost without thinking about them. For this reason, you need to practice repeating the motion process of Soul Link until you know exactly what you are doing, and until it is one smooth movement. The necessary feelings of connection will work when you know the process and make it feel natural, so that you can pretty much do the rubbing motion without thinking. Make it smooth and easy, remembering to "feel" all the connections you can. Work with NO memory in any way until the process is smooth and complete.

Even now, stop and see if you can do this as one fluid movement. It needs to begin with a full contact slide all the way past the palm, then a glide over, and all the way back. Sense all the feelings of connection as you create a continuous flowing motion. The speed will ultimately come down to approximately ten seconds for four complete passes on this hand.

In the beginning your goal is just one continuous process of feeling all you can with the connective slide and glide process of Soul Link. Today it takes trained clients twenty seconds or less to complete the process. It is not about the speed; it is about the contact and the feeling of making contact happen naturally and automatically.

\section{Doing the healing}

Now, pick a 2015 negative memory out of a one to ten range, with ten being the worst, in the three to four range. Be sure it gives you negative emotions and feelings ONLY in the range of a three to four as you learn the process. As you think about this memory, have some fun and play it in your head so that you can learn everything you can about it. Notice the sharpness, clarity, words, sounds, and feelings. What can you distinguish so that when you play it again later, you can check for differences? This will help with the quality of your first experience with Soul Link.

When you have learned all you can about this memory, place your right hand finger tips against your left hand where you know the little finger is in line with the top knuckles. Now turn your focus as much as possible on all you recall as you repeat this memory while completing four passes on each hand. Remember to do four passes on both hands. A key component at this time is focusing your attention on the memory and not on how to move your hands. All hand movement should be close to automatic for best results.

When you are done, allow yourself to stop thinking about what you were doing and simply look around wherever you are and internally tell yourself some of the things you see around you. This causes you to stop thinking about the memory on which you were working.

Check to discover what has happened to the emotions and feelings that caused you to choose this memory in the first place. Is there anything there now? Are the feelings neutral? If there is anything remaining for negative feeling and emotions, run the full pattern again as you pay attention to the emotions and feelings that now arise. I can tell you, however, with a three to four level memory, it is not normal for there to be residual that remains unless you paid more attention to the touch process than the memory content. Initially you may have a tendency to do that because you are just learning.

Now check the memory. Try to note any changes that you can find to determine if this memory is still there and intact just as you remembered it before. Some will have a hard time finding the memory, and for some it may seem gone.

Give yourself another Soul Link test using another memory ONLY in the three to four ranges with ten being the worst.

Congratulations! You have learned to "Heal Memories in Seconds!" $" 10$

NOTE: As mentioned earlier, for the sake of this presentation, this research is presented as taught to a client. There is another complete set of protocols that are taught in Soul Link Certification that allow any therapist to do the work to the client. For the sake of your understanding and testing, this material was presented only in this format.

Where we go from here and the multiple other uses, that are life changing in "seconds," are taught in Soul Link Certification. You worked on memories in the three to four range. With more experience using Soul Link, you will move up in your scale of memory healing. Gary personally, in his first year of presenting Soul Link, worked with 1,700 clients at a "worst memory ever" and PTSD level with $95 \%$ success with first time use on the memory. For those where he has only done the protocols to them the same results have been shown.

Soul Link is already being used to resolve fears, upsets, anger, guilt, self-sabotage, blocks to success, etc. while shown to improve academic and athletic performance, self-confidence, self-esteem, health and wellness and more.

\section{Research chart}

Note: This chart shows the success when doing Soul Link for the first and second time on the emotions and feelings from the first minor and first major memory.

\section{Time period June 2014 - August 2015 (Chart 1).}

It should also be noted that every person in the 2,200 was being taught for the first time, so that any reason for secondary gain to not neutralize that memory may not have been taken into consideration by them at that time. Thus, the memory was checked and reported by them as neutralized.

\section{Conclusion}

Therapists all over the globe work to help people with PTSD. The NET-CE September 2015 Continuing Education Review Course on PTSD states, "The overall objectives of PTSD therapy is to treat the four core symptom clusters of

\section{Intrusive re-experiencing}

\section{Avoidance}

3. Negative alterations in cognitions and mood and

4. Hyperarousal 
Psychotherapy is the backbone of PTSD therapy with Pharmacotherapy used as an adjunct if necessary. ${ }^{11}$

The modalities currently in use today are at best $60 \%$ to $80 \%$ successful with a substantial financial burden associated because of the time and energy spent in therapeutic sessions along with the cost of the medications.

Since 1981 Gary has been teaching clients to neutralize negative memories successfully, in groups or one-on-one. Gary feels strongly that is it time for proper research and documentation that will allow for a scientific analysis of this and other aspects of his work. When his two day full program, called Restoration, can leave a client with no past memory they can immediately remember that still bothers them, then what else can be learned from his 35years of work?

Humanity needs help to handle the traumas associated with this life. There are no current double-blind studies to support this work. Perhaps you, amongst others which Gary is consulting with, will be presenting case reports in the near future as a larger randomized study becomes organized and potentially funded. At 71, Gary would tell you, "I would not be alive had I not learned to understand 'life as energy.' I should have been dead at least twenty times, and yet that calling to help others has continued to pull these resources forward to you, and I am still here to help!"

This partial work as presented here is only to help you understand the potential use of Soul Link. It is protected by law and may not be used for any form of financial gain or research by you or others with whom you share this research without the express written permission of Gary Sinclair.

\section{Conflicts of interest}

Being the owner and copyright holder of this work, Gary has a financial interest in the future of how it is ultimately researched and shared. Although it is now part of a basic certification training course, there is much to be done in the field of research as it relates to Epigenetics.

\section{Acknowledgments}

None.

\section{Funding}

None.

\section{References}

1. Ravitz MD, Leonard J. Application of the Electrodynamic Field Theory in Biology, Psychiatry, Medicine and Hypnosis. American Journal of Clinical Hypnosis. 1959;1(4):135-150.

2. Myss, Caroline. Anatomy of the Spirit. New York, USA: Random House; 1996. p. 33-34

3. Hoffman, Enid. HUNA: A Beginner's Guide. Whitford Press, Atglen, Pennyslvania, USA. 1981. pp.146-147.

4. Quillin, Patrick. Healing Secrets from the Bible. Tulsa, Oklahoma, USA: The Nutrition Times Press; 1996. p. 68

5. Rollin McCrary, McCraty's book, The Energetic Heart

6. Mike Atkinson, Raymond Trevor Bradley, Rollin McCraty. Electrophysiological Evidence of Intuition: Part 1. The Surprising Role of the Heart. Journal of Alternative and Complementary Medicine. 2004;10(1):133-143.

7. White, John Future Science. Anchor Books, New York, USA, p. 97.

8. Burmeister, Alice, Monte, et al. The Touch of Healing. Bantam Books, New York, USA. 1997. p. 43.

9. Eden, Donna. Energy Medicine. New York, USA: Penguin Putnam; 1998. p. 47.

10. Gary Sinclair. Healing Memories in Seconds. USA: Waterside Publication, Cardiff; 2015.

11. NET-CE - Continuing Education Review Course on PTSD. 2015. 